\title{
Can Disks Produce Companions by Gravitational Fragmentation?
}

\author{
Richard H. Durisen \\ Department of Astronomy, Swain West 319, Indiana University, \\ Bloomington IN 47405, U.S.A.
}

\begin{abstract}
The nonlinear outcome of gravitational instabilities in disks depends critically on the thermal physics of the gas. Under conditions where thermal energy is lost efficiently, disks disrupt into dense arms, arclets, and clumps. However, the evidence about whether clumps can ever become permanent bound objects is currently inconclusive. Under conditions where cooling is less efficient or where a balance between heating and cooling is achieved, the amplitudes reached by gravitational instabilities are relatively modest. The result is disk heating and transport of mass and angular momentum rather than condensation of bound companions. Future numerical simulations need to resolve the disk vertical structure and include more realistic equations of state and energy transport.
\end{abstract}

\section{Why Ask the Question?}

It has been known since the 1960's and 70's that the self-gravity of thin disks can drive instabilities when the Toomre stability parameter $Q$ is sufficiently small (cf. Toomre 1981, Binney \& Tremaine 1987). For gas disks, $Q=c_{s} \kappa / \pi G \Sigma$, where $c_{s}$ is the sound speed, $\kappa$ is the epicyclic frequency of the disk, $G$ is the gravitational constant, and $\Sigma$ is the disk surface density. Gas pressure, represented by $c_{s}$, stabilizes short wavelengths; and disk rotation, represented by $\kappa$, stabilizes long wavelengths. For $Q<1$, self-gravity, represented by $\Sigma$, destabilizes the disk; and ringlike axisymmetric perturbations grow.

Along a cooling sequence of decreasing $c_{s}$ and hence $Q$, a disk will encounter nonaxisymmetric instabilities somewhat before the ringlike ones. This occurs when $Q<1.5$ to 2 or so, depending on the detailed structure of the disk. The wavelength of the most unstable perturbation scales as the surface density of the disk. So it is reasonable to suggest that companions to stars, particularly gas giant planets, might be able to form from massive disks through gravitational condensation (Kuiper 1951, Cameron 1978), in much the same way that star formation is mediated by the Jeans' instability. The absence of a compelling theory for formation of close binaries, difficulties with the core-accretion model of gas giant planet formation, and the discovery of massive substellar companions in orbits close to their parent stars all serve to renew interest in the possibility of companion formation via gravitational fragmentation of a disk. 


\section{Recent Results}

\subsection{The Dynamic 90's}

The nonaxisymmetric gravitational instabilities which occur when $Q \lesssim 1.5$ are self-stimulated and dynamic, i.e., they produce spiral disturbances which grow spontaneously from low-amplitude noise with growth time scales of about a rotation period. As summarized in the review by Papaloizou \& Lin (1995), there are a variety of mechanisms and conditions which can contribute to growth, as well as a variety of possible outcomes. Perhaps the best understood growth mechanism is that a leading spiral wave amplifies as it "swings" into a trailing spiral wave near corotation. If a feedback loop can be established which sends leading waves back into the amplifier with the right phase, this will produce sustained growth.

Several studies in the early 1990's (e.g., Papaloizou \& Savonije 1991, Laughlin \& Bodenheimer 1994) showed that gravitational instabilities can produce strong transient spiral waves which transport mass and angular momentum through gravitational torques but do not produce permanent condensations. This idea was reinforced in a series of papers culminating in Laughlin, Korchagin, \& Adams (1998) which demonstrated by simulations and by linear and nonlinear analyses that, for polytropic equations of state (EOS's), the saturation amplitude of unstable modes is determined by nonlinear mode interaction. The amplitudes achieved are well below levels required for fragmentation, but large enough to produce strong torques. Meanwhile, using a thin-disk particle code and a crude treatment of heating and cooling, Tomley et al. $(1991,1994)$ demonstrated that heating by dissipation of mode energy balanced by cooling could also regulate the steady-state amplitude of multi-armed gravitational instabilities and produce sustained mass and angular momentum transport.

Other analyses early in the decade suggested that the dominant mechanism for instability of a massive disk might be SLING, in which a one-armed spiral grows through displacement of the central star and the disk mass to opposite sides of the system barycenter (Adams, Ruden, \& Shu 1989, Shu et al. 1990). A global, lopsided instability of this type might be much more likely to form a companion object from the disk. An SPH disk simulation by Adams \& Benz (1992) did show growth of a one-armed spiral with a mass condensation forming at the disk outer edge.

All of these studies involved some simplifications, such as point-mass stars, thin-disk approximations, polytropic EOS's, artificial inner and outer boundary conditions, and/or other special assumptions. Several questions remained. Particularly, what mechanisms really prevail in determining the nature and nonlinear amplitude of the instabilities, and does disk fragmentation occur under any conditions?

\subsection{Thermal Physics Rules}

The set of 3D hydrodynamics simulations presented by Pickett et al. (1998, 2000a; hereafter PCDL) were undertaken with the goal of testing the role of thermal physics in regulating gravitational instabilities. These calculations are unique to date in that they resolve the star, the star/disk interface, and the disk vertical structure. The disk is small (equatorial radius $R_{e q}=0.1 \mathrm{AU}$ ) 
and massive (disk mass $M_{d}=0.2 M_{\odot}$ ) and not much larger (stellar radius $R_{*}=$ $0.1 R_{e q}$ ) or less massive (stellar mass $M_{*}=0.3 M_{\odot}$ ) than the star. This numerical star/disk model is a 3D counterpart to the analytic thin-disk models of Cassen \& Moosman (1981). Whether such a state might actually be achieved during star formation is certainly debatable, but it provides a numerical laboratory in which PCDL could vary the EOS while making few other artificial assumptions.

In PCDL, the disk thermal structure is initially set to be vertically isentropic with a specific entropy that decreases radially outward so that $Q \approx 1.5$ over most of the disk. The 3D hydrodynamics simulations are performed with a second-order finite-difference scheme using a fixed cylindrical grid. Four different assumptions are made about the behavior of the gas as the disk evolves: 1.) Locally Isothermal (ISO). The disk temperature remains fixed in space at its initial value. 2.) Locally Isentropic (ISE). The specific entropy of the gas remains fixed spatially. 3.) Adiabatic (ADI). The specific entropy of fluid elements remains constant as they move. 4.) Adiabatic with Artificial Viscosity (ADIAV). Artificial viscosity is added to the momentum and energy equations to represent friction and heating due to irreversible processes, such as shocks. In all four cases, the star is initially isentropic and is evolved adiabatically. Note that, because of the radial temperature gradient in the disk, inward moving fluid elements heat up and outward moving fluid elements cool down automatically for the ISO assumption. As artificial as the ISO and ISE cases may seem, they are the most common EOS assumptions for disk simulations in the literature. Although cooling is not explicitly included, energy is effectively lost by the disk in all cases but ADIAV, with the ISO case exhibiting the most extreme losses. In all cases, multi-armed spirals form quickly near the star/disk boundary; but, after a little more than one outer rotation, a dominant two-armed spiral takes over in the disk.

The nonlinear outcomes are radically different for different EOS's. For the ISO case, the spiral structure increases in amplitude to the limit of resolution. This causes complete disruption of the disk into dense arms, arclets, and transient clumps. Some of the arms expand to nearly twice the initial disk outer radius after only two outer disk rotations. The logarithmic greyscale of the equatorial density in the left panel of Figure 1 shows that typical densities in the filamentary structure are similar to that of the central star! I deliberately say that the disk is "disrupted" rather than "fragmented", because no persistent bound fragments seem to form. Clumps appear and disappear in typically a quarter to half an orbit period. At the other extreme, for the ADIAV case, the two-armed spiral never becomes very strong; and heating dramatically thickens the vertical structure of the disk. Results for ISE and ADI are similar to each other and intermediate between the extremes. As shown in the right panel of Figure 1 for the ADI case, the two-armed spiral becomes saturated at an amplitude which does not disrupt the disk but causes mass and angular momentum transport. The ISE and ADI are similar to the polytropic disks studied by Laughlin et al. (1998), and nonlinear mode interaction may well be the operative mechanism for saturation in these cases.

The overall conclusions from the PCDL experiments are that thermal physics of the gas determines the nonlinear amplitude of gravitational instabilities and that, even under the assumption of extremely efficient cooling, as repre- 

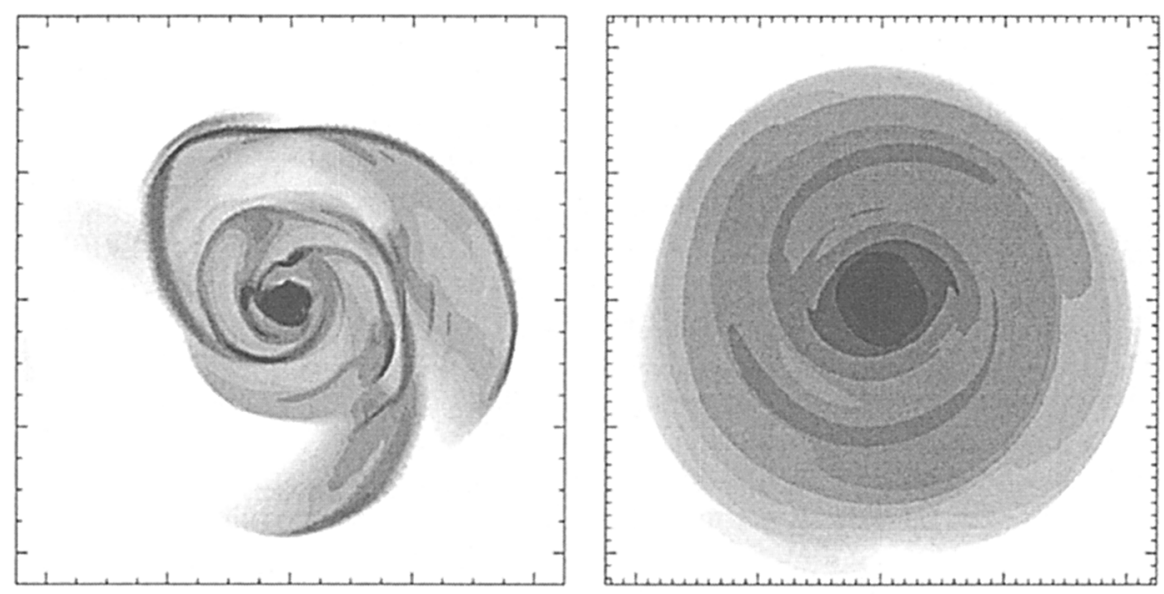

Figure 1. Logarithmic greyscales of the equatorial plane density for two PCDL simulations. The left panel shows The ISO case after almost two outer rotations; the right panel shows the ADI case after three outer rotations. The dimension of the left panel is twice that of the right panel. In the ADI case, the outer radius of the disk does not change appreciably. The central star is represented by black. The sense of rotation here and in Figures 2 and 3 is counterclockwise.

sented by the ISO case, there is no evidence for formation of bound fragments during the two to three outer rotations spanned by the simulations. Although nonlinear mode interactions lead to some lopsided behavior, a two-armed spiral is always the dominant mode that grows in the disk, not a one-armed SLINGdriven mode. Many of the qualitative features of the locally isothermal PCDL calculations are confirmed by Nelson et al. (1998), except that they do see clump formation in their SPH simulations.

\subsection{To GGPP or Not to GGPP?}

At the same time as the PCDL and Nelson et al. work was being done, Boss (1997, 1998) produced 3D simulations of massive Solar Nebula models. Typically, his 1998 models have disk radii between 0.5 and $10 \mathrm{AU}, M_{d}=0.13 M_{\odot}$, a fairly flat overall $\Sigma(r)$, and $M_{*}=M_{\odot}$. The inner regions are hot and have high $Q$-values which decrease outward; the outer few AU's have $Q$-values of order unity. His unstable models, which mostly utilize the same EOS as the ISO case in PCDL, readily produce one or more dense condensations with masses typically several times the mass of Jupiter $\left(M_{J}\right)$. The objects last for many consecutive orbits and satisfy various tests of boundedness, included being well above the local Jeans mass and being tidally stable. With this work, Boss revived the idea that giant gaseous protoplanets (GGPP's) might form directly from cold massive disks.

Boss's results are radically at odds with PCDL. In the Boss calculations, the clumps grow slowly in place over many rotation periods in a relatively quiescent manner. A major computational difference between Boss and PCDL is that Boss 

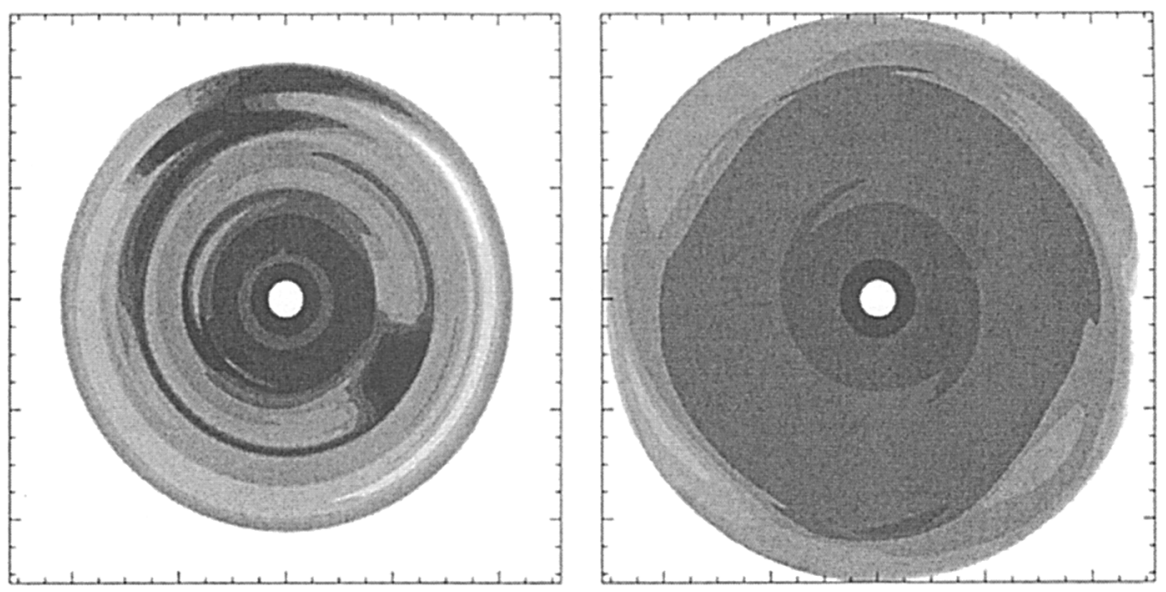

Figure 2. Logarithmic greyscales of the equatorial plane density after 180 yrs (6.4 outer rotations) for two simulations with the Pickett et al. Solar Nebula model. The left panel is the ISOB case; the right panel, the ADIAV case. The clump at about 4 o'clock in the lefthand panel persists for many orbits and has the mass of a brown dwarf.

damps velocities of radial and vertical expansion in his calculations for numerical convenience. To check Boss's results, Pickett et al. $(2000 \mathrm{~b}, \mathrm{c})$ generated an initial $3 \mathrm{D}$ equilibrium state which closely resembles the Boss Solar Nebula. It has inner and outer radii of 0.76 and $10 \mathrm{AU}, M_{d}=0.13 M_{\odot}$, a similar $\Sigma(r)$, and a fixed central mass $M_{*}=M_{\odot} . Q$ smoothly decreases from values of 3 to 10 in the inner region to about 1.1 over an annulus near the edge which is $1.3 \mathrm{AU}$ wide. Three comparison simulations were computed with different EOS's and velocity assumptions : 1.) ISOB, locally isothermal with Boss-like damping of expansion velocities; 2.) ISO, locally isothermal with free expansion; 3.) ADIAV, adiabatic with artificial viscosity and free expansion.

The left panel of Figure 2 shows the endstate of the ISOB calculation. After about five outer rotations, a dense clump forms and grows in place at 5.4 AU. It persists for more than 3.5 orbits, with no sign of dissipating, and accumulates $30 M_{J}$ by the end. This image is similar to Figure 15 in Boss (1998). The ISO simulation behaves quite differently and in essential agreement with PCDL. Instabilities grow more rapidly; and, by about two outer rotations, dense thin spiral arcs of gas expand off the computational grid. Apparently, Boss velocity damping suppresses the natural expansion of the disk due to gravitational torques, an essential nonlinear feature of the instability. Suppression of this response allows relatively gentle growth of massive condensations. The right panel of Figure 2 shows the ADIAV endstate from Pickett et al.. Heating by dissipation of mode energy prevents formation of dense clumps or arclets. The spiral structure and expansion is much more modest than for the ISO case.

While Pickett et al. were computing these comparisons, Boss (2000) removed velocity controls from his calculations and now finds behavior similar to PCDL. An endstate from one of his recent ISO simulations is shown in the left panel of Figure 3. The disk now extends from 4 to $20 \mathrm{AU}$ and has a mass of 0.09 

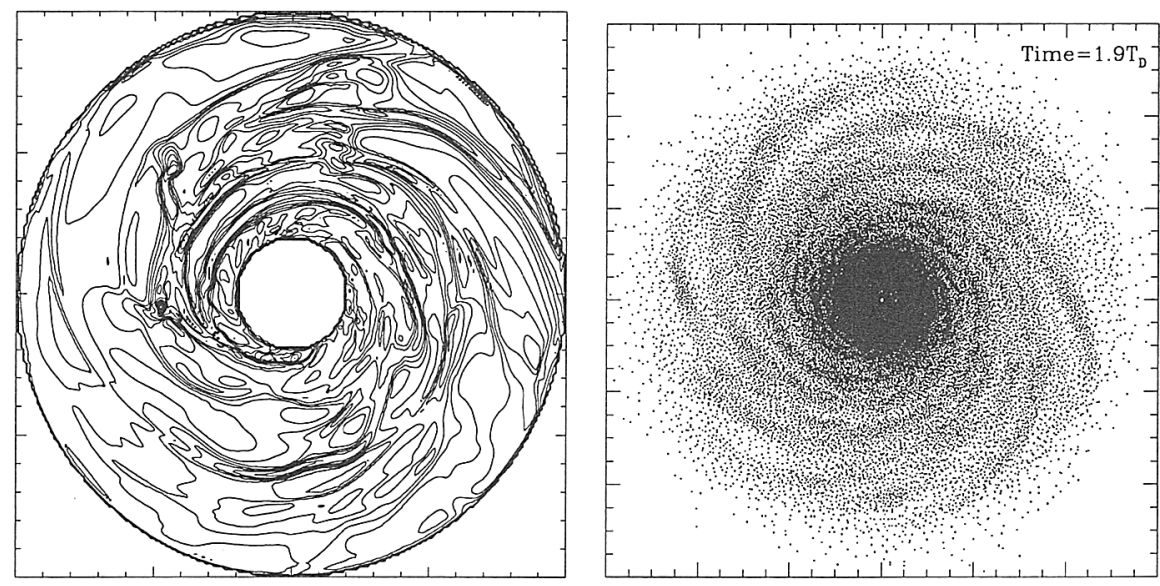

Figure 3. Left Panel: Logarithmic contour plot of the equatorial plane density in a Boss (2000) Solar Nebula calculation after 374 yrs. The dense spot at 9 o'clock is a clump of $5 M_{J}$ which persists for two orbits then dissolves. The edge of the panel spans 40 AU. Right Panel: SPH particle positions in the equatorial plane for the "free thermodynamics" simulation B2m4 in NBR. The edge of the panel spans $100 \mathrm{AU} . T_{D}$ is about 500 yrs.

$M_{\odot}$. Note the generally similar morphology of this disrupted disk to the PCDL ISO case in Figure 1. Boss (2000) actually presents a series of calculations in which the resolution of the hydrodynamic grid and of the gravitational potential representation are increased when dense structure appears. The density of the densest clumps increases as resolution increases. Without velocity damping, the disk disruption is violent and chaotic, as in PCDL, with dense clumps appearing and disappearing. With considerably higher resolution than used by PCDL, Boss does find that one multi- $M_{J}$ clump, shown as the dense, small concentration of contours near 9 o'clock in Figure 3, is able to persist for two complete orbits. However, even this feature dissolves back into the ISO maelstrom after two orbits, despite being apparently bound by a Jeans-mass criterion.

\subsection{Heating, Cooling, and the Third Spatial Dimension}

If the outcome of gravitational instabilities is so sensitive to the thermal physics of the gas, then the necessary direction for future research is to follow Tomley et al. (1991, 1994) and do simulations which include modeling of the gas thermodynamics, including relevant heating and cooling mechanisms. A long, new stride down this path has been taken by Nelson, Benz, \& Ruzmaikina (2000, hereafter NBR).

Although the NBR SPH simulations resolve only $r$ and $\phi$, they are two and a half dimensional in the sense that a separate computation is done of radiative equilibrium in the $z$-direction along with the thin-disk hydrodynamics. The disks are larger, extending out to 50 or $100 \mathrm{AU}$, and are better resolved than in Nelson et al. (1998). Here, artificial viscosity is introduced to represent 

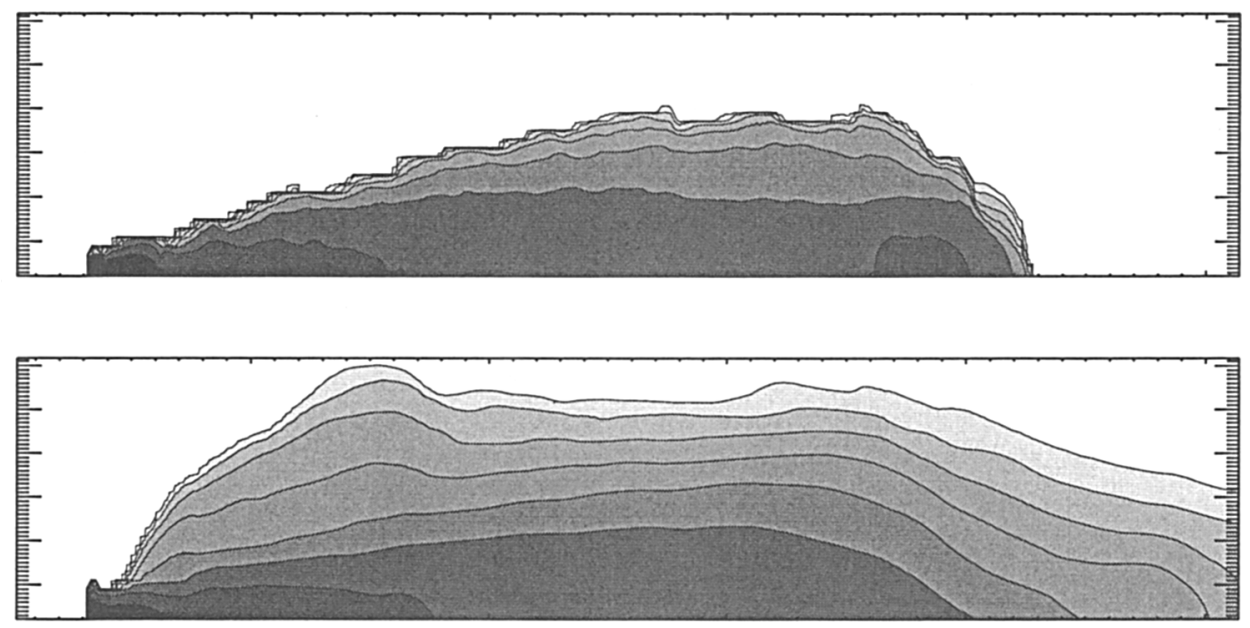

Figure 4. Greyscale representation of the azimuthally averaged density in the $(r, z)$ plane for the Pickett et al. Solar Nebula. The top panel is the initial $10 \mathrm{AU}$-radius disk. The bottom panel is the ADIAV case at 180 yrs (6.4 outer disk rotations). The greyscale levels are the same as in Figure 2.

both shock dissipation and an underlying heating by the disk's shear. NBR also include radiative cooling, under optically thick conditions in the inner disk and optically thin in the outer disk. Nelson refers to this as "free thermodynamics", because the disk is permitted to seek its own balance among heating, cooling, and instability. It in fact does so within about two rotations. As shown in the right panel of Figure 3, the sustained spiral structure can be strong in the outer, optically thin part of the disk but is well below the extreme amplitudes of an ISO evolution.

If the physics of heating and cooling does regulate gravitational instabilities, then fully 3D calculations need to become the norm. As shown in Figure 4, the heating due to dissipation of spiral waves for the ADIAV case of Pickett et al. (2000c) is disproportionately large in the upper layers of the disk, in the sense that the heating rate there represents a large fraction of the local thermal energy within one orbit period. This causes dramatic thickening of the vertical structure, as shown in Figure 4. Even though $z$ is not explicitly resolved in their calculations, NBR also report that the vertical structure of their inner disks, as measured by a photospheric condition, becomes quite distended. NBR note that, although they do not include the effect, disk surface heating by the central star can be a key component in the energy balance and appearance of the disk (e.g., D'Alessio et al. 1998). So shadowing by a thickened inner disk could have profound and observable consequences.

Important insights often come from semi-analytic analyses. Following work by Korycansky \& Pringle (1995) on axisymmetric perturbations, Lubow \& Ogilvie (1997) studied the form of spiral waves driven by external forcing in thick polytropic disks. They found that the spiral waves are then predominantly $\mathrm{f}$ modes and that these modes concentrate energy toward the disk surface. PCDL 
presented arguments for similar characteristics of their self-stimulated waves. In another study, Cassen \& Woolum (1996) considered radiative damping of spiral waves propagating in thin disks by using a simple model for radiative equilibrium in the $z$-direction. With self-stimulated waves, the sign of the effect might reverse. Future analyses of this type will provide guidance in efforts to understand the behavior of complex numerical simulations.

\subsection{Resisting Temptation}

There is, admittedly, a seductive quality to the disk disruptions seen in ISO simulations. It is tempting to conclude that, sooner or later, something will form out of the dense, chaotic, dynamic structure. However, even if the unstable disk region is optically thin initially, the objects whose formation we wish to witness, GGPP's, brown dwarfs, or stars, are themselves optically thick. For instance, using a Pollack et al. (1994) Rosseland mean opacity of about $0.2 \mathrm{~cm}^{2} / \mathrm{g}$ at $40 \mathrm{~K}$, the dense clump in the left panel of Figure 3 has a normal optical depth of a few times $10^{5}$ ! Clumps in the ISO simulations with free expansion originate as intersections, kinks, or collisions points between filaments. So the formation times associated with dense structures in ISO calculations are much shorter than the orbital time scale. The filaments themselves must effectively radiate thermal energy rapidly as they form. Taken together, the rapid formation of optically thick structure suggests that there must come a point in a GGPPforming calculation when isothermality breaks down, even if valid initially. The only way to be certain that any clump will become a permanent, bound object is to follow the thermal physics in detail. ISO calculations cannot, in themselves, demonstrate companion formation; they can only suggest the possibility. In fact, the outer half of the disks in the NBR simulations are optically thin; yet, with free thermodynamics, NBR report that they "rarely" produce dense clumps, nevermind permanent ones.

\section{Past, Present, and Future}

\subsection{Conclusions to Date}

- Gas thermal physics controls the nonlinear outcome of gravitational instabilities in disks.

- Disk disruption into dense structure occurs only for the extreme thermodynamics of a nearly isothermal gas response.

- Even in this case, dense clumps appear to be transient when the natural expansion of the disk due to gravitational torques is allowed to occur.

- When heating, cooling, and instability mechanisms achieve balance, spiral waves tend to saturate at modest amplitudes and produce sustained transport of mass and angular momentum.

- Resolution of the vertical structure is necessary to treat the effects of wave dissipation correctly. 


\subsection{Proceed with Caution!}

From now on, when confronted with calculations which purport to show disk fragmentation and formation of bound objects, for instance in a cold circumbinary disk or through disk collisions or through perturbations by an existing companion, we must expect authors to discuss whether their numerical resolution is adequate and whether their assumptions about thermal physics are realistic. Isothermal calculations will be particularly suspect, because they are strongly biased toward disk disruption. Authors should show that, for the application at hand, any thermal energy which would have been produced under realistic thermodynamics by shock heating or compression could in fact be radiated away as quickly as isothermality effectively assumes. Even then, authors should demonstrate that the clumping is not caused by inadequate numerical resolution (Truelove et al. 1997) and that clumps persist for very many orbits. Except perhaps for efforts to understand an isolated process or mechanism in principle, we should declare that the days of disk simulations with simplistic gas physics are now over. For realistic applications, the energy budget and EOS must be treated with something approaching the same fidelity as the hydrodynamics.

\subsection{Is there Hope?}

Results to date may be inconclusive; but they suggest that a number of conditions are conducive to disk disruption and hence increase the chances of companion formation: i) $Q \lesssim 1.5$, ii) a nearly isothermal disk response, iii) confinement of instability to a narrow region, and perhaps iv) mechanisms which counteract the natural tendency of the disk to expand. Requirement (i) means either an early phase of disk evolution, when the disk is more likely to be massive, or a local accumulation of material due to the failure of other disk transport and heating mechanisms (e.g., Gammie 1996). Condition (ii) can be satisfied in two ways, either by cooling and radiative diffusion times which are short compared with an orbital period, as is generally the case in the outer disk, or by the presence of a major phase transition which flattens the temperature response of the disk. Condition (iii) might limit the severity of the disk response, but the numerical evidence to date is that gravitational instabilities quickly infect the entire disk, even regions of high $Q$. With regard to (iv), little can be said at present; but it might be interesting to explore the effect of tidal truncation of the disk by a pre-existing companion. Finally, for GGPP's at least, a hybrid picture may eventually emerge where disk self-gravity accelerates core accretion (Boss 2000, Wuchterl, Guillot, \& Lissauer 2000).

All the cautions from the previous section apply, but it will be interesting to see where the increasing complexity and verisimilitude of numerical simulations will eventually take us. We live in exciting times. We are finally on the verge of being able to put credible physics into Solar Nebula-scale dynamical models. We are fortunate that this ability dovetails with the growing realization that we absolutely have to!

Acknowledgments. I would like to thank A.P. Boss, P. Cassen, A.F. Nelson, and B.K. Pickett for their help with preparation of this chapter. The author is supported in part by a grant from NASA's Origins of Solar Systems Progam. 


\section{References}

Adams, F. C., \& Benz, W. 1992, in IAU Colloq. 135, Approaches to Double and Multiple Star Research, ed. H. A. McAlister \& W. I. Hartkopf (San Francisco: ASP), 185

Adams, F. C., Ruden, S. P., \& Shu, F. H. 1989, ApJ, 347, 959

Binney, J., \& Tremaine, S. 1987, Galactic Dynamics (Princeton Univ. Press: Princeton)

Boss, A. P. 1997, Science, 276, 1836

Boss, A. P. 1998, ApJ, 503, 923

Boss, A. P. 2000, ApJL, in press

Cameron, A. G. W. 1978, Moon Planets, 18, 5

Cassen, P., \& Moosman, A. 1981, Icarus, 48, 353

Cassen, P., \& Woolum, D. 1996, ApJ, 472, 789

D'Alessio, P., Canto, J., Calvet, N., \& Lizano, S. 1998 ApJ, 500, 411

Gammie, C. F. 1996, ApJ, 457, 355

Korycansky, D. G., \& Pringle, J. E. 1995, MNRAS, 272, 618

Kuiper, G. P. 1951, PNAS, 37, 1

Laughlin, G., \& Bodenheimer, P. 1994, ApJ, 436, 335

Laughlin, G., Korchagin, V., \& Adams, F. C. 1998, ApJ, 504, 945

Lubow, S. H., \& Ogilvie, G. I. 1998, ApJ, 504, 983

Nelson, A. F., Benz, W., Adams, F. C., \& Arnett, D. 1998, ApJ, 502, 342

Nelson, A. F., Benz, W., \& Ruzmaikina, T. V. 2000, ApJ, 529, 357

Papaloizou, J. C. B., \& Lin, D. N. C. 1995, ARA\&A, 33, 505

Papaloizou, J. C. B., \& Savonije 1991, MNRAS, 248, 353

Pickett, B. K., Cassen, P., Durisen, R. H., \& Link, R. 1998, ApJ, 504, 468

Pickett, B. K., Cassen, P., Durisen, R. H., \& Link, R. 2000a, ApJ, 529, 1034 \& Erratum ApJ, 530, 1106

Pickett, B. K., Durisen, R. H., Cassen, P., \& Mejia, A. C. 2000b, in IAU Symp. 200, Poster Proceedings, ed. B. Reipurth \& H. Zinnecker (Potsdam), 190

Pickett, B. K., Durisen, R. H., Cassen, P., \& Mejia, A. C. 2000c, ApJL, subm.

Pollack, J. B., Hollenbach, D., Beckwith, S., Simonelli, D. P., Roush, T., \& Fong, W. 1994, ApJ, 421, 615

Shu, F. H., Tremaine, S., Adams, F. C., \& Ruden, S. P. 1990, ApJ, 358, 495

Tomley, L., Cassen, P., \& Steiman-Cameron, T. Y. 1991, ApJ, 382, 530

Tomley, L., Steiman-Cameron, T. Y., \& Cassen, P. 1994, ApJ, 422, 850

Toomre, A. 1981, in The Structure and Evolution of Normal Galaxies, ed. S. M. Fall \& D. Lynden-Bell (Cambridge: Cambridge Univ. Press), 111

Truelove, J. K., Klein, R. I., McKee, C. F., Holliman, J. H., Howell, L. H., \& Greenough, J. A. 1997, ApJL, 489, L179

Wuchterl, G., Guillot, T., \& Lissauer, J. J. 2000, in Protostars \& Planets IV, ed. V. Mannings, A. P. Boss \& S. S. Russell (Tucson: Univ. Arizona Press), in press 